\title{
Shorter TR Combination with Finer Atlas Positively Modulate Topological Organization of Brain Networks: A Resting State fMRI Study
}

\section{Yan Zhang}

China Jiliang University

\section{QILI HU}

Fifth People's Hospital of Shanghai Fudan University

Jiali Liang

Zhejiang University School of Medicine Second Affiliated Hospital

Zhenghui Hu

Zhejiang University of Technology

\section{Tianyi Qian}

Siemens Healthcare China

Kuncheng Li

Xuanwu Hospital Department of Radiology

Xiaohu Zhao ( $\nabla$ xhzhao999@263.net)

Shanghai Fifth People's Hospital of Fudan University https://orcid.org/0000-0002-3630-0879

Peipeng Liang

Capital Normal University

\section{Research article}

Keywords: Magnetic Resonance Imaging, fMRI, Brain, Atlas, Frequency

Posted Date: December 2nd, 2020

DOI: https://doi.org/10.21203/rs.3.rs-116032/v1

License: (c) (1) This work is licensed under a Creative Commons Attribution 4.0 International License. Read Full License 


\section{Shorter TR Combination with Finer Atlas \\ Positively Modulate Topological Organization of Brain Networks: A Resting State fMRI Study}

1 YAN ZHANG ${ }^{1, \S}$, QILI HU ${ }^{2, \S}$, JiALI LIANG ${ }^{3}$, ZHENGHUI HU $^{4}$, TIANYI QIAN ${ }^{5}$, KUNCHENG

$2 \mathrm{LI}^{6,7}, \mathrm{XIAOHU} \mathrm{ZHAO}^{2, *,}$, PeIPENG LIANG $^{8, *,}$,

$3{ }^{1}$ College of Optical and Electronic Technology, China Jiliang University, Hangzhou,

4 China

$5 \quad{ }^{2}$ Department of Imaging, The Fifth People's Hospital of Shanghai, Fudan University,

6 Shanghai, China

$7 \quad{ }^{3}$ The Second Affiliated Hospital, Zhejiang University School of Medicine, Hangzhou,

8 China

$9{ }^{4}$ Center for Optics and Optoelectronics Research, College of Science, Zhejiang

10 University of Technology, Hangzhou, China

$11{ }^{5}$ MR Collaboration, Siemens Healthcare China, Beijing, China

$12{ }^{6}$ Department of Radiology, Xuanwu Hospital, Capital Medical University, Beijing,

13 China

$14 \quad{ }^{7}$ Beijing Key Lab of MRI and Brain Informatics, Beijing, China

$15{ }^{8}$ School of Psychology, Capital Normal University, Beijing, China

$17{ }^{\S}$ Author contributions

18 Yan Zhang and Qili Hu contributed equally to this work and are first author. Xiaohu

19 Zhao and Peipeng Liang contributed equally to this work and are correspondent

20 authors

21 * Correspondent author

22 Xiaohu Zhao

23 E mail: xhzhao999@263.net

24 Phone: 86-021-24289092

25 Peipeng Liang

26 E mail: ppliang@cnu.edu.cn

27 Phone: 86-13552053752 


\section{Abstract}

32 Background: The simultaneous multislice echo planar imaging technique can shorten

33 the repetition time (TR) of blood oxygen level-dependent acquisition and thus

34 acquires more information. However, little is known about the influence of higher temporal resolution on functional networks. Whether the topological organization of small-world networks is modulated in the multispectra at high temporal resolution is still unclear. Results: The network reconstruction based on the shorter TR and the finer atlas, showed significant ( $p<0.05$, Bonferroni correction) increases in normalized clustering coefficient, small-worldness, clustering coefficient, local efficiency and

40 global efficiency, and reductions in normalized characteristic path length and

41 characteristic path length. Conclusions: The shorter TR coupled with the finer atlas can positively modulate topological characteristics of brain networks. Although five multispectra present properties of small-world networks, the properties of the network in $0.082-0.1 \mathrm{~Hz}$ are weaker than those in $0.01-0.082 \mathrm{~Hz}$. These findings provide new insights into the topological patterns of brain networks and have implications for the study of brain connectomes and their applications in brain disease. 


\section{Background}

53 The brain network of human has been proven to consist of small-world networks according to the characterization of graph theory $[1,2]$. The topology of networks can be described by different measures, for example, node degree, hub, etc. Particularly, two parameters, the clustering coefficient $(\mathrm{Cp})$ and the characteristic path length $(\mathrm{Lp})$, are frequently adopted to investigate properties of a network. Specifically, smallworld networks have both a high $\mathrm{Cp}$ and a short Lp, showing a good local efficiency (Eloc) and a high global efficiency (Eg) [3]. imaging (fMRI) studies. Typically, a 2 3 s TR may result in the signal being obscured by mixing physiological noise into the neuronal-induced BOLD fMRI signal [4]. To partially filter these undesirable signals, a higher temporal resolution may be a feasible way. However, the conventional EPI sequence still needs several seconds to cover the whole brain. Recently, a simultaneous multislice (SMS) EPI technique has been proposed that can shorten TR without reduction in echo time (TE) and signal-to-noise ratio [5]. However, little is known about the influence of shorter TR on properties of

73 the brain network.

The scale of the brain parcellation is known to have an effect on the organization of brain networks [6]. The properties of networks vary with the distinct parcellation 
scales [7]. The Automated Anatomical Labeling (AAL) atlas [8] is one of the most popular brain atlases. By contrast, the parcellation containing 200 regions [9] was adopted. It remains unclear whether there is an interaction between the temporal resolution and the scale of brain parcellation.

(1)
2

Additionally, previous studies have indicated that brain networks exhibit various properties in distinct frequency bands $[10,11]$. Particularly, the subtle temporal differences might be averaged out within the typical frequency interval [12], which might lead to information being overlooked. It is necessary to decompose the typical frequency interval into several subbands to further evaluate the organization of networks. 8

There are two main aims in this study. First, we inspected the effects of TR length (0.5 vs. $2 \mathrm{~s}$ ) and the scale of brain parcellation (90 vs. 200 regions of interest (ROIs)), as well as their possible interaction, on the characteristics of brain networks. Second, we tested whether the topological organization of networks was modulated in the multispectra when the BOLD signal was acquired at a high temporal resolution.

\section{Results}

\subsection{Single spectrum}

ANOVA results showed that the main effect of brain parcellation was significant as revealed by Cp, Lp, Eloc, and Eg, as well as $\lambda$. The main effect of TR length was significant, as revealed by Eloc, $\gamma$ and $\sigma$. There was no interaction effect between them. The detailed statistical results are shown in Table 1. 


\subsection{Simple main effects of brain parcellation}

102 When networks were constructed based on the AAL atlas, the results revealed that the dataset with the shorter TR possessed significantly larger $\gamma$, Cp, and Eloc (Fig. 1).
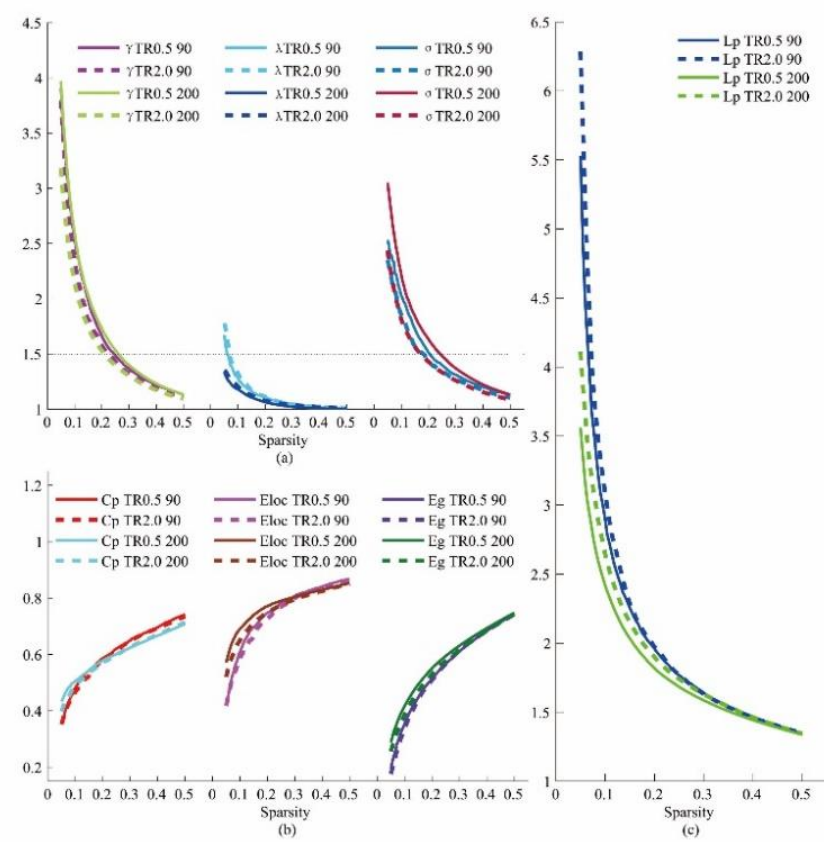

104

105 Fig.1. Network metrics for a single spectrum (0.01-0.1 Hz). All measures were represented as functions

106 of sparsity across a range from 0.05 to 0.5 with an increment of 0.0045 . TR0.5 means the repetition

107 time is $0.5 \mathrm{~ms}$, and the last digit presents the number of regions.

108 When networks were built based on a finer atlas, the shorter TR condition showed

109 significant increases in $\gamma, \sigma, \mathrm{Cp}$, Eloc and $\mathrm{Eg}$ as well as decreases in $\lambda$ and Lp. The

110 statistical comparisons of the different brain parcellation methods showed that the

111 finer atlas condition had significant decreases in $\lambda, \mathrm{Cp}, \mathrm{Lp}$, and Eloc and an increase

112 in Eg over a wide range of sparsity, in both TR conditions. 
114 The subfrequency band of $0.064-0.082 \mathrm{~Hz}$ was chosen as an illustration to replicate

115 the findings from the single spectrum, as shown in Fig. 2.
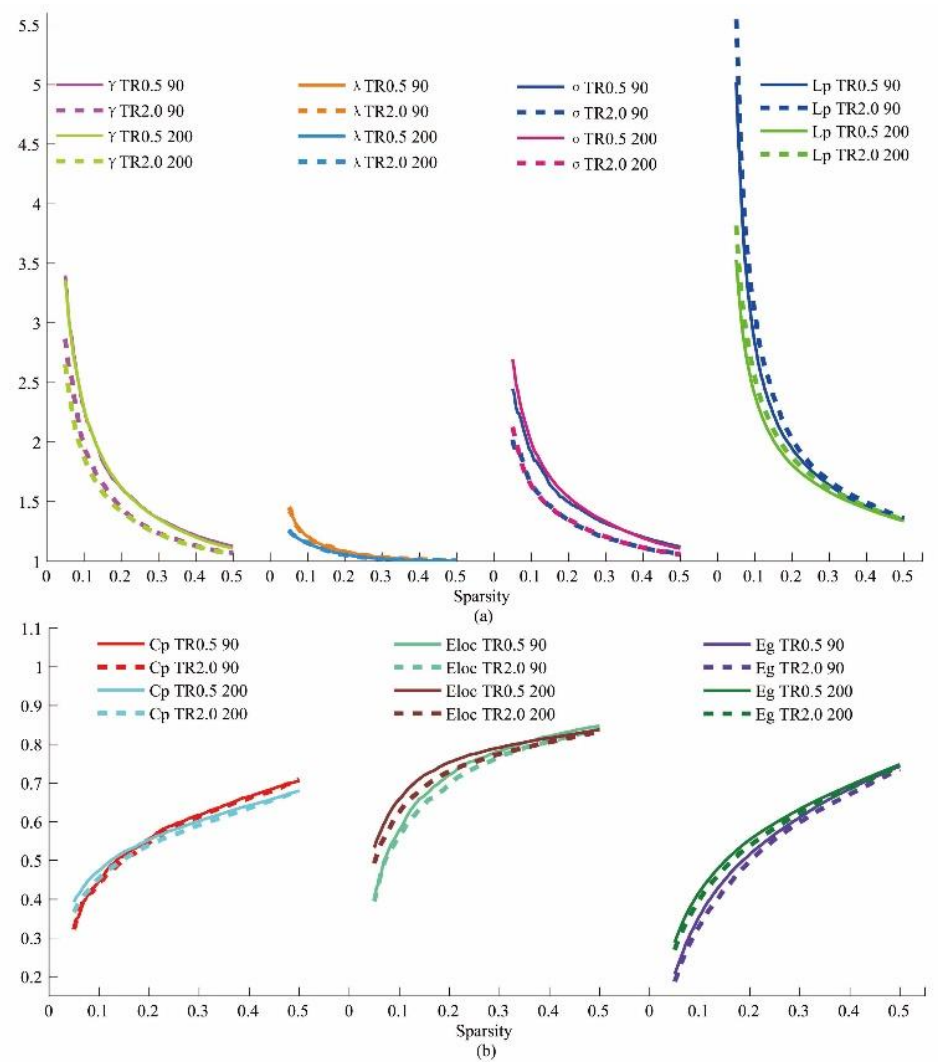

Fig.2. Network metrics for a particular subspectrum $(0.064-0.082 \mathrm{~Hz})$.

119 When the AAL atlas was used, the shorter TR condition showed significant increases

120 in $\gamma, \sigma$, Eloc, and Eg and decreases in $\lambda$ and Lp. When the high-resolution atlas was

121 applied, the shorter TR condition showed significant increases in $\gamma, \sigma, \mathrm{Cp}$, and Eloc

122 and decreases in Lp. In the comparison of the two different parcellation methods, the

123 results revealed that the finer atlas presented significant reductions in $\lambda, \mathrm{Lp}$, and

124 increases in $\mathrm{Cp}$, Eloc, Eg. These results were totally applied to the other sub-

125 spectrums. 
128 All five multispectra presented the properties of small-world networks (Fig. 3);

129 however, their characteristics were different from those of the single spectrum. In

130 particular, $0.082-0.1 \mathrm{~Hz}$ presented significant differences in all seven parameters. In

131 the $0.01-0.046 \mathrm{~Hz}$ spectra, $\gamma, \sigma, \mathrm{Cp}$, and Eloc were significantly decreased, and $\lambda$ in

$132 \quad 0.046-0.082 \mathrm{~Hz}$ was significantly reduced.
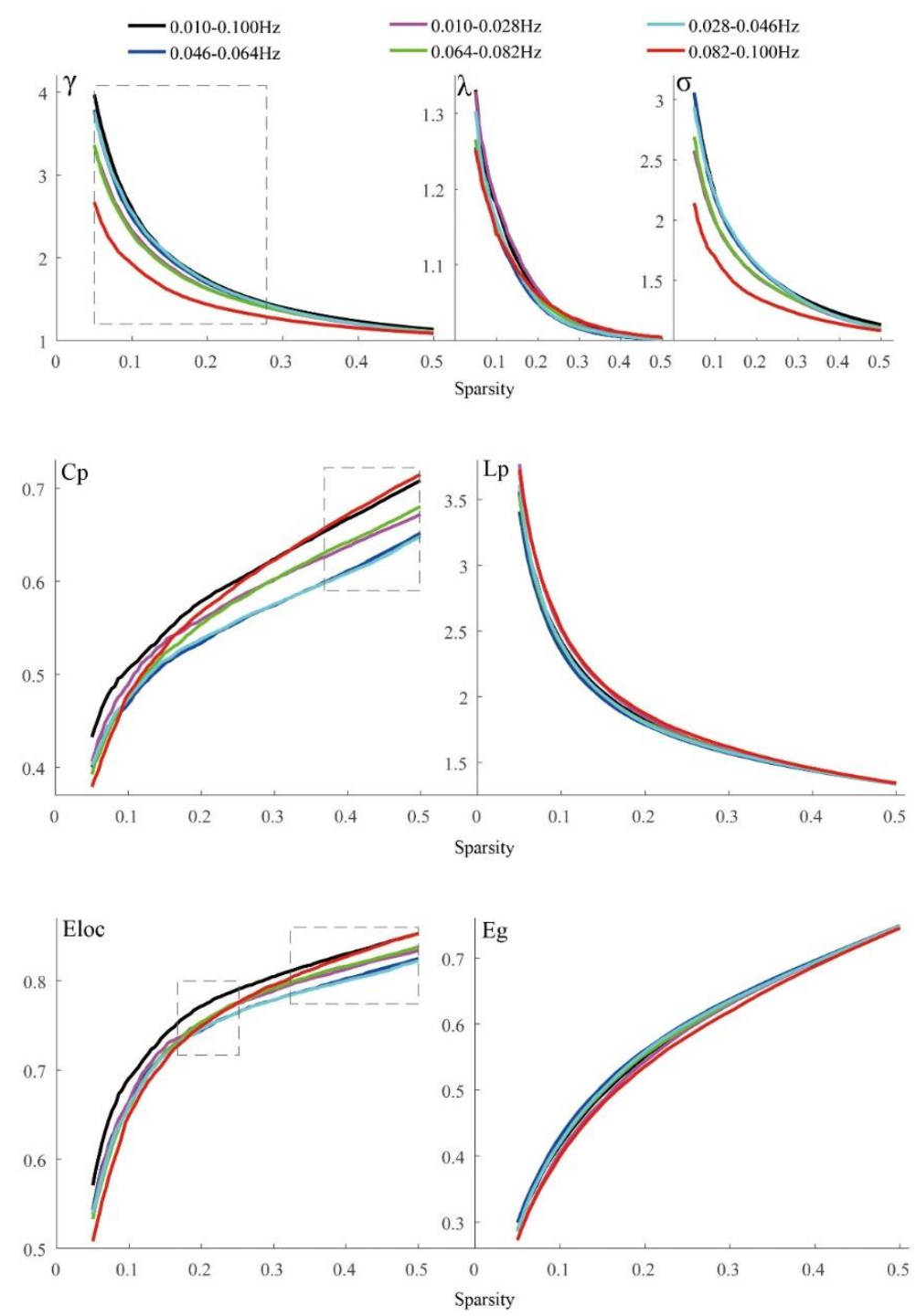

134 Fig.3. Network metrics for the multispectra at the global level. 
135 The pairwise comparisons between the five subfrequency bands showed that the two

136 parameters $\sigma$ and $\mathrm{Eg}$ of $0.082-0.1 \mathrm{~Hz}$ were significantly smaller than those of the

137 other four multispectra, while Cp, Lp, and Eloc of 0.082-0.1 Hz were larger than

138 those of the other four multispectra. Cp of $0.01-0.028 \mathrm{~Hz}$ was significantly larger than

139 that of $0.028-0.082 \mathrm{~Hz}, \mathrm{Lp}$ and $\mathrm{Eg}$ of $0.01-0.028 \mathrm{~Hz}$ were significantly longer and

140 lower than those of $0.046-0.064 \mathrm{~Hz}$. Eloc of $0.064-0.082 \mathrm{~Hz}$ was significantly higher

141 than that of $0.028-0.046 \mathrm{~Hz}$.

\section{Discussion}

143 The results indicated that the shorter TR can modulate the properties of small-world

144 networks. This was directly supported by the fact that the $\sigma$ of the shorter TR

145 conditions was significantly larger than that of the longer TR conditions.

146 Additionally, it was found that the Cp, Lp, Eloc, and Eg from the shorter TR

147 conditions were tighter, shorter, higher, and larger than those of the longer TR

148 conditions, respectively.

150 Our results revealed that the organization of the network is changed as a function of

151 the parcellation, as characterized by the significant differences in $\lambda, \mathrm{Cp}, \mathrm{Lp}$, Eloc and

152 Eg between the two parcellation schemes. In particular, networks based on a finer

153 parcellation exhibited different small-worldness. This was highly in accordance with a

154 previous study [6].

155

156 Based on 90 ROIs, the significant differences between the two TR lengths existed

157 only in the parameters of $\gamma, \mathrm{Cp}$ and Eloc. Based on 200 ROIs, significant differences

158 between the two TR lengths were found in all seven parameters. Particularly, the 
159 shorter TR coupled with the finer parcellation, might be more beneficial to the

160 transmission of information and trend toward reinforcing the optimal balance between

161 local specialization and global integration in a small-world model.

162

163 The topological properties of the network of $0.082-0.1 \mathrm{~Hz}$ were slightly weaker

164 than those of the other four subfrequency bands. This is completely congruent with

165 some previous reports. For example, they $[10,16]$ found that small-world properties

166 were salient at lower frequencies. However, there was a counterexample [17], which

167 reported that the highest small-worldness was located on scale 2. It was argued that

168 this inconsistency can be accounted for by the difference in the number of time points.

169 Limited number of time points may lose some important information.

170

1714 Conclusion

172 This study first demonstrated that the BOLD signal acquired with SMS-EPI at a

173 higher temporal resolution, coupled with the finer atlas, can positively modulate the

174 small-world topological properties of brain networks. Although five multispectra

175 present properties of small-world networks, the properties of the network in $0.082-0.1$

$176 \mathrm{~Hz}$ are weaker than those in $0.01-0.082 \mathrm{~Hz}$.

177

178 


\section{Materials and Methods}

180

181

182

183

184

185

186

187

188

189

190

191

192

193

194

195

196

197

198

199

200

\subsection{Subjects}

Twenty healthy young volunteers were recruited (aged 23-28 years, mean age $=25.3$

years, ten male). The exclusion criteria were (1) a history of neurological or

psychiatric disorder or head injury; (2) current treatment with vasoactive or

psychotropic medication; (3) any physical or intellectual disability; and (4) any

contraindication to MRI. Data from one subject was discarded due to a failure to

cooperate during the MRI scan.

\subsection{Data acquisition}

MRI scans were acquired using a MAGNETOM Verio 3T MR Scanner (Siemens,

Erlangen, Germany). The resting-state fMRI (R-fMRI) data were obtained using two scanning protocols: (1) shorter TR protocol: TR, $500 \mathrm{~ms}$; TE, $30 \mathrm{~ms}$; flip angle (FA), $90^{\circ} ; 32$ slices; matrix, 64*64; voxel size, $3.75 * 3.75 * 4 \mathrm{~mm}^{3}$; SMS factor, 4; (2) longer TR protocol: TR, 2000 ms; TE, 30 ms; FA, 90³ 32 slices; matrix, 64*64; voxel size, $3.75 * 3.75 * 4 \mathrm{~mm}^{3}$; SMS factor, 1 . During the R-fMRI scans, subjects were instructed to hold still, keep their eyes closed.

\subsection{Data Preprocessing}

All images were preprocessed using the Statistical Parametric Mapping (SPM12, http://www.fil. ion.ucl.ac.uk/spm/) software package and Data Processing Assistant for Resting-State fMRI (DPARSF) [13]. The first 10 time points of the longer TR data and the first 20 time points of the shorter TR data were discarded. The images were then corrected for the staggered order of slice acquisition and head motion. Data from 
201 one subject were excluded due to excess head motion (translation $>2 \mathrm{~mm}$ ). The data

202

203

204

were then spatially normalized to the Montreal Neurological Institute space and resampled to $3 \mathrm{~mm}$ isotropic voxels using the normalization parameters estimated during unified segmentation [14]. Subsequently, the images were smoothed with a Gaussian kernel of $4 * 4 * 4 \mathrm{~mm}^{3}$ full-width at half-maximum. Temporal filtering $(0.01$ $\mathrm{Hz}<\mathrm{f}<0.1 \mathrm{~Hz}$ ) was applied to the time series.

Since the R-fMRI signals are complex, the frequency interval $(0.01-0.1 \mathrm{~Hz})$ was decomposed into five equal-length spectra. The five spectra were $0.01-0.028 \mathrm{~Hz}$, 0.028-0.046 Hz, 0.046-0.064 Hz, 0.064-0.082 Hz, and 0.082-0.1 Hz. Regressions of six motion parameters, white matter signals and cerebrospinal fluid signals were performed. The global signal was not regressed out. Finally, two atlases (AAL and Craddock template) were adopted to extract the BOLD time series.

\subsection{Network Analysis}

\subsubsection{Contruct the correlation matrix}

The functional connectivity matrices were obtained by calculating Pearson correlation coefficients between each pair of ROIs before a Fisher's $z$ transformation was done. Then, the matrices were put into the GRETNA toolbox [15] to compute the parameters of networks.

\subsubsection{Thresholding}

The measurement of sparsity was defined as a percentage of the existing number of edges divided by the maximal possible number of edges in a network. The range of 
$0.05<$ sparsity $<0.5$ with an increment of 0.0045 was employed to threshold the correlation matrices into binary matrices.

\subsubsection{Network metrics}

226 For a given binary graph $G$ with $N$ nodes and $K$ edges, the Cp of the network is

227 defined as

$$
\mathrm{C}_{\mathrm{p}}=\frac{1}{\mathrm{~N}} \sum_{\mathrm{i} \in \mathrm{G}} \mathrm{C}_{\mathrm{i}}(1)
$$

229 where $C_{i}$ is the ratio of the number of existing connections to the number of all 230 possible connections in a subgraph. $C_{i}=2 * E_{i} /\left[D_{i}\left(D_{i}-1\right)\right]$, where $D_{i}$ represents the 231 degree (defined as the number of edges connected to the node) of the $i$ th node, and $\mathrm{E}_{\mathrm{i}}$ 232 denotes the number of edges in the subgraph $\mathrm{G}_{\mathrm{i}}$ (defined as a set of nodes directly 233 connecting to the $i$ th node). The $\mathrm{L}_{\mathrm{p}}$ is defined as the mean of the shortest path length 234 of all pairs of nodes in the network:

$$
\mathrm{L}_{\mathrm{p}}=\frac{1}{\mathrm{~N}(\mathrm{~N}-1)} \sum_{\mathrm{i}, \mathrm{j} \in \mathrm{G}} 1_{\mathrm{ij}}
$$

236 where $\mathrm{l}_{\mathrm{ij}}$ expresses the shortest path between node $i$ and $j$. The Eloc can be calculated 237 as follows:

$$
\mathrm{E}_{\mathrm{loc}}=\frac{1}{\mathrm{~N}} \sum_{\mathrm{i} \in \mathrm{G}} \mathrm{E}_{\mathrm{loc}}(\mathrm{i})
$$

239 where Eloc(i) is the local efficiency of node $i$. The Eloc(i) can be measured as

$240 \quad \mathrm{E}_{\mathrm{loc}}(\mathrm{i})=\frac{1}{\mathrm{~N}_{\mathrm{G}_{\mathrm{i}}}\left(\mathrm{N}_{\mathrm{G}_{\mathrm{i}}}-1\right)} \sum_{\mathrm{j}, \mathrm{k} \in \mathrm{G}_{\mathrm{i}}} \frac{1}{\mathrm{j}_{\mathrm{jk}}}$, where $\mathrm{N}_{\mathrm{G}_{\mathrm{i}}}$ is the number of nodes in the subgraph $\mathrm{G}_{\mathrm{i}}$. The

241 Eg of the network can be formalized as follows:

$$
\mathrm{E}_{\mathrm{g}}=\frac{1}{\mathrm{~N}(\mathrm{~N}-1)} \sum_{\mathrm{i} \neq \mathrm{j} \in \mathrm{G}} \frac{1}{\mathrm{l}_{\mathrm{ij}}}
$$


243 The normalized clustering coefficient $\gamma=C p / C r a n d$ and normalized characteristic path

244 length $\lambda=L p / L r a n d$ were also calculated, where Crand and Lrand represent the mean

245 of clustering coefficient and the average of characteristic path length of random

246 networks, respectively. Two-way analysis of variance (ANOVA) and two-sample t-

247 test were used to measure significant differences ( $p<0.05$, Bonferroni correction).

\section{Abbreviations}

250 TR, repetitioin time;

251 SMS, simultanenous multislice;

$252 \gamma$, normalized clustering coefficient;

$253 \lambda$, nornalized characteristic path length;

$254 \sigma$, small-worldness;

255 Cp, clustering coefficient;

256 Lp, characteristic path length;

257 Eloc, local efficiency;

258 Eg, global efficiency;

259 BOLD, blood oxygen level-dependent;

260 R-fMRI, resting-state functional magnetic resonance imaging;

261 AAL, automated anatomical labeling;

263 Declarations

264 Ethics approval and consent to participate: This study was conducted in

265 accordance with the Declaration of Helsinki. Informed consent to participate in the 266 study was obtained from the participants verbally and in writing. This study was 
approved by the Ethical Review Board of Shanghai Fifth People's Hospital of Fudan University.

269 Consent for publication: Not applicable.

270 Availability of data and materials: The datasets used and/or analysed during the

271 current study are available from the corresponding author on reasonable request.

272 Competing interests : The authors declare that they have no competing interests.

273 Funding: This study was funded by the Natural Science Foundation of China (grant 274 number_81271552), and the Science and Technology Commission of Shanghai

275 Municipality (grant number_18411970300), and the Health Industry Clinical

276 Research of the Shanghai Health and Family Planning Committee (grant number 277 _201840018)

278 Authors' contributions: XZ: Conceptualization, PL: Conceptualization, JL:

279 Methodology, ZH: Formal analysis and investigation, TQ: Data Curation, QH:

280 Writing - original draft preparation, YZ: Writing - review and editing, QL: Funding 281 acquisition. All authors read and approved the final manuscript.

282 Acknowledgements: We thank American Journal Experts (AJE) for the English 283 language editing of this manuscript.

\section{Reference}

286 1. Watts DJ and Strogatz SH (1998) Collective dynamics of 'small-world' networks.

287 Nature 393(6684):440-442

288 2. Park CH, Kim SY, Kim YH et al. (2008) Comparison of the small-world topology

289 between anatomical and functional connectivity in the human brain. Physica a-

290 Statistical Mechanics and Its Applications 387(23):5958-5962 
291 3. Xia MR and He Y (2011) Magnetic Resonance Imaging and Graph Theoretical 292 Analysis of Complex Brain Networks in Neuropsychiatric Disorders. Brain

293 Connectivity 1(5):349-365

294 4. Cordes D, Haughton VM, Arfanakis K et al. (2001) Frequencies Contributing to 295 Functional Connectivity in the Cerebral Cortex in "Resting-state" Data. AJNR.

296 American journal of neuroradiology 22(7):1326-1333

297 5. Preibisch C, Castrillon JG, Buhrer M et al. (2015) Evaluation of Multiband EPI 298 Acquisitions for Resting State fMRI. Plos One 10(9):e0136961

299 6. Wang JH, Wang L, Zang YF et al. (2009) Parcellation-Dependent Small-World 300 Brain Functional Netw orks: A Resting-State fMRI Study. Human Brain Mapping $30130(5): 1511-1523$

302 7. Zalesky A, Fornito A, Harding IH et al. (2010) Whole-brain anatomical networks:

303 Does the choice of nodes matter? Neuroimage 50(3):970-983

304 8. Tzourio-Mazoyer N, Landeau B, Papathanassiou D et al. (2002) Automated 305 Anatomical Labeling of Activations in SPM Using a Macroscopic Anatomical 306 Parcellation of the MNI MRI Single-Subject Brain. Neuroimage 15(1):273-289 307 9. Craddock RC, James GA, Holtzheimer PE et al. (2012) A whole brain fMRI atlas 308 generated via spatially constrained spectral clustering. Human Brain Mapping 309 33(8):1914-1928

310 10. Achard S, Salvador R, Whitcher B et al. (2006) A Resilient, Low-Frequency, 311 Small-World Human Brain Functional Network with Highly Connected Association 312 Cortical Hubs. The Journal of Neuroscience 26(1):63-72

313 11. Liang X, Wang JH, Yan CG et al. (2012) Effects of Different Correlation Metrics 314 and Preprocessing Factors on Small-World Brain Functional Networks: A Resting315 State Functional MRI Study. Plos One 7(3):e32766 
316 12. Wee CY, Yap PT, Denny K et al. (2012) Resting-State Multi-Spectrum Functional

317 Connectivity Networks for Identification of MCI Patients. Plos One 7(5):e37828

318 13. Yan CG and Zang YF (2010) DPARSF: A MATLAB Toolbox for "Pipeline" Data

319 Analysis of Resting-State fMRI. Frontiers in systems neuroscience 4(13):13

320 14. Ashburner J and Friston KJ (2005) Unified segmentation. Neuroimage 26(3):839-

$321 \quad 851$

322 15. Wang JH, Wang XD, Xia MR et al. (2015) GRETNA: a graph theoretical network 323 analysis toolbox for imaging connectomics. Frontiers in Human Neuroscience 9:458

324 16. Supekar K, Menon V, Rubin D et al. (2008) Network Analysis of Intrinsic

325 Functional Brain Connectivity in Alzheimers Disease. PLoS computational biology

326 4(6):e1000100

327 17. Fornito A, Zalesky A, Bassett DS et al. (2011) Genetic Influences on Cost-

328 Efficient Organization of Human Cortical Functional Networks. Journal of

329 Neuroscience 31(9):3261-3270

330

331

332

333

334

335

336

337

338

339

340 


\begin{tabular}{|c|c|c|c|c|c|c|c|c|c|}
\hline & \multicolumn{3}{|c|}{ The main effect of brain parcellation } & \multicolumn{3}{|c|}{ The main effect of TR length } & \multicolumn{3}{|c|}{ Interaction effect } \\
\hline & Sparsity & F Value & $P$ Value & Sparsity & F Value & $P$ Value & Sparsity & F Value & $P$ Value \\
\hline $\mathrm{Cp}$ & $\begin{array}{l}0.05-0.095 \\
0.2705-0.5\end{array}$ & $\begin{array}{l}7.8420- \\
74.3617\end{array}$ & $\begin{array}{l}1.6201 \times 10^{-12}- \\
0.0066\end{array}$ & I & l & I & l & l & l \\
\hline Lp & $0.05-0.239$ & $\begin{array}{l}7.7083- \\
35.6278\end{array}$ & $\begin{array}{l}9.6360 \times 10^{-8}{ }_{-} \\
0.0071\end{array}$ & l & l & l & l & I & l \\
\hline Eloc & $\begin{array}{l}0.05-0.212 \\
0.374,0.3785 \\
0.3875-0.5\end{array}$ & $\begin{array}{l}7.9918- \\
149.1590\end{array}$ & $\begin{array}{l}8.2742 \times 10^{-19} \\
0.0062\end{array}$ & $\begin{array}{l}0.0635,0.0086,0.095 \\
0.1085,0.1175-0.1535 \\
0.176,0.1895-0.1985\end{array}$ & $7.7136-11.1360$ & $0.0014-0.0071$ & l & l & l \\
\hline Eg & $0.05-0.2615$ & $\begin{array}{l}7.9212- \\
55.2858\end{array}$ & $\begin{array}{l}2.3183 \times 10^{-10} \\
0.0064\end{array}$ & l & I & l & I & l & / \\
\hline$\gamma$ & I & I & l & $0.455-0.5$ & $7.6964-8.6072$ & $0.0046-0.0071$ & I & I & l \\
\hline$\lambda$ & $0.05-0.257$ & $\begin{array}{l}8.0123- \\
20.9622\end{array}$ & $\begin{array}{l}2.0513 \times 10^{-5}- \\
0.0061\end{array}$ & l & l & l & l & I & l \\
\hline$\sigma$ & l & l & l & $0.4595-0.5$ & $7.9814-8.4051$ & $0.0050-0.0062$ & l & l & I \\
\hline
\end{tabular}


Figures
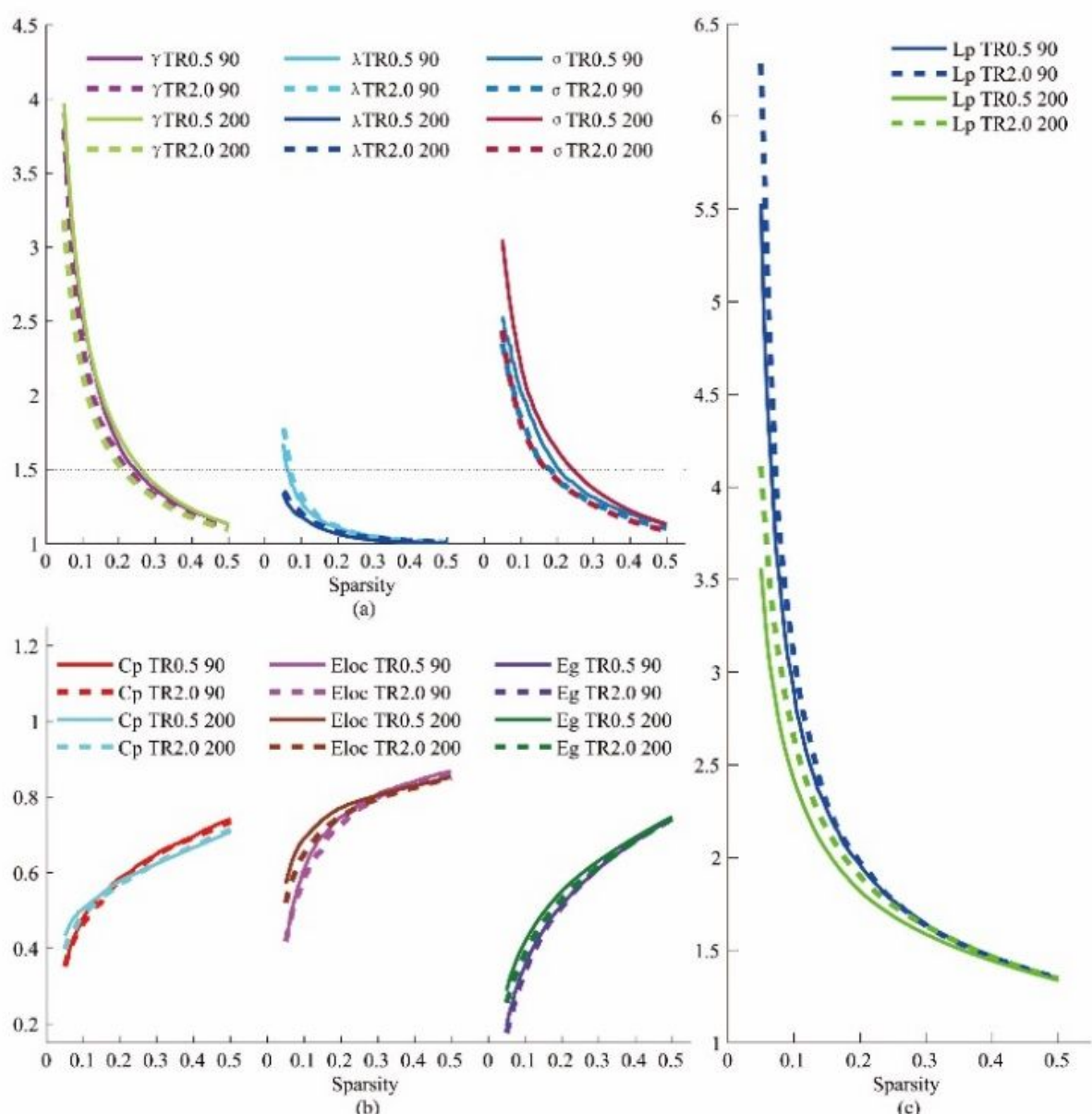

\section{Figure 1}

Network metrics for a single spectrum $(0.01-0.1 \mathrm{~Hz})$. All measures were represented as functions of sparsity across a range from 0.05 to 0.5 with an increment of 0.0045 . TR0.5 means the repetition time is $0.5 \mathrm{~ms}$, and the last digit presents the number of regions. 

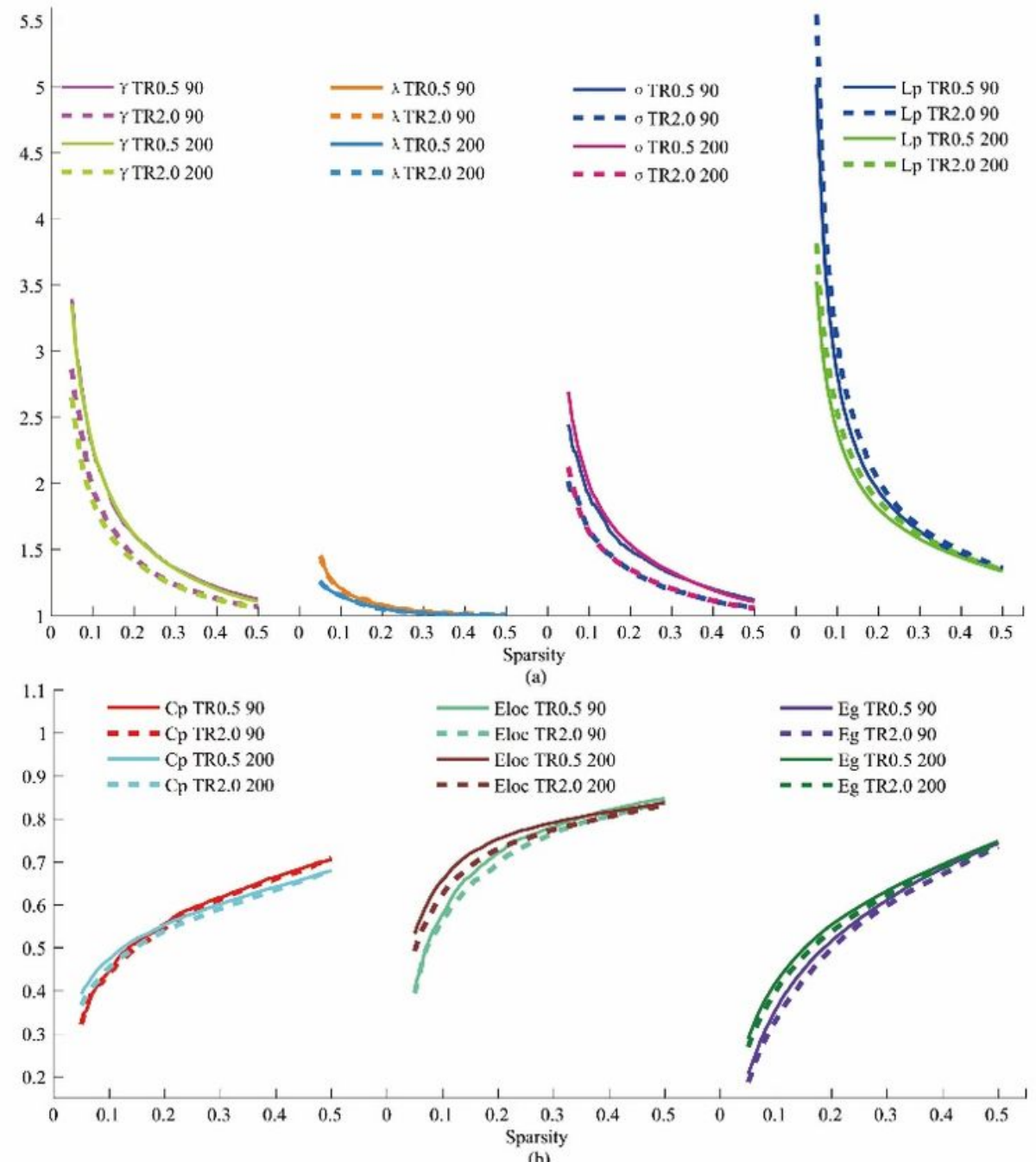

(b)

Figure 2

Network metrics for a particular subspectrum $(0.064-0.082 \mathrm{~Hz})$. 

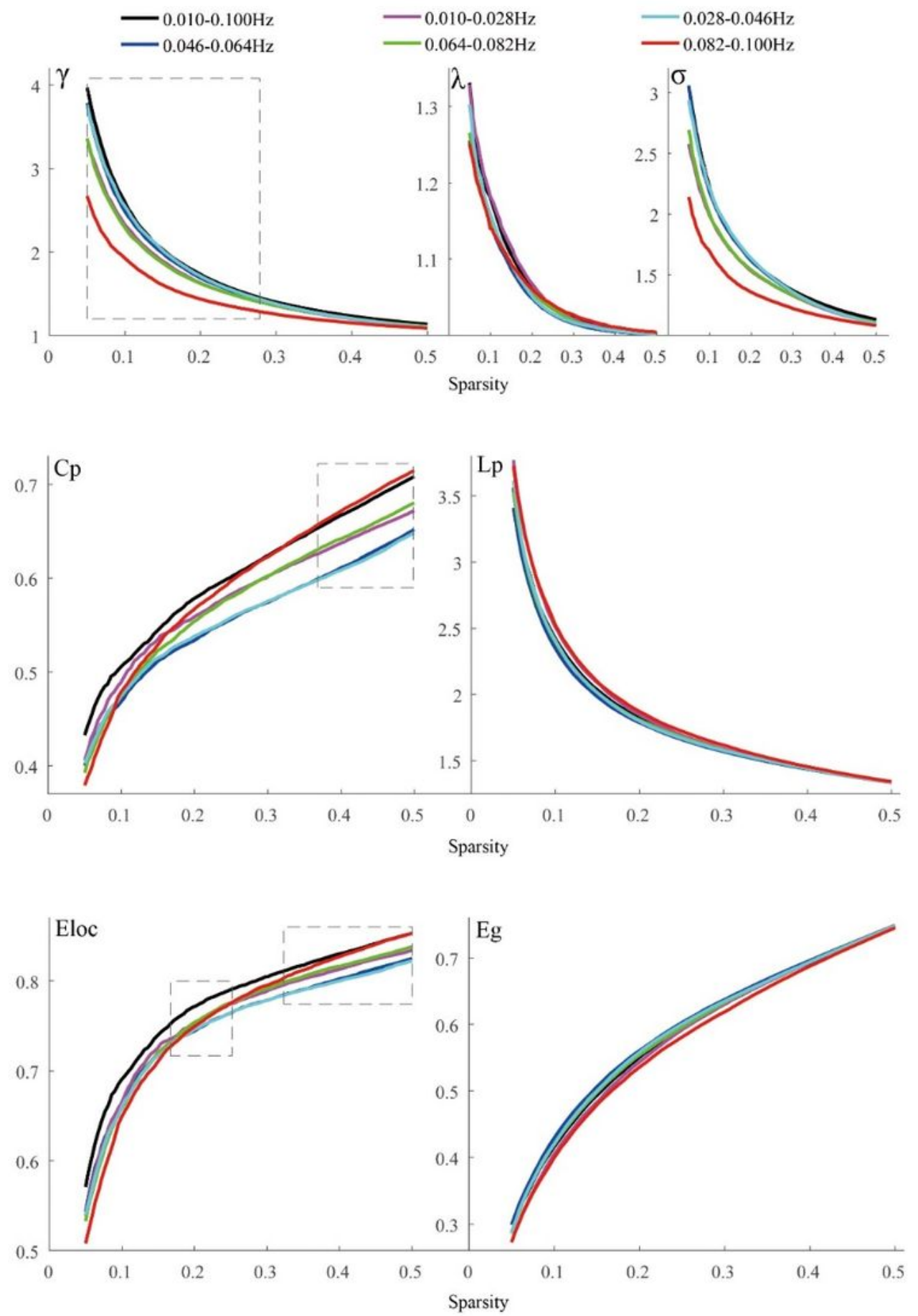

Figure 3

Network metrics for the multispectra at the global level. 